\title{
The use of tibial tuberosity-trochlear groove indices based on joint size in lower limb evaluation
}

\author{
Peter Wilhelm Ferlic ${ }^{1}$ (D) Armin Runer ${ }^{1} \cdot$ Florian Dirisamer $^{2} \cdot$ Peter Balcarek $^{3}$. \\ Johannes Giesinger ${ }^{4} \cdot$ Rainer Biedermann ${ }^{1} \cdot$ Michael Christian Liebensteiner ${ }^{1}$
}

Received: 31 January 2017 / Accepted: 1 June 2017 /Published online: 19 June 2017

(C) The Author(s) 2017. This article is an open access publication

\begin{abstract}
Purpose The correlation between tibial tuberosity-trochlear groove distance (TT-TG) and joint size, taking into account several different parameters of knee joint size as well as lower limb dimensions, is evaluated in order to assess whether TT-TG indices should be used in instead of absolute TT-TG values. Methods This study comprised a retrospective analysis of knee CT scans, including 36 cases with patellofemoral instability (PFI) and 30 controls. Besides TT-TG, five measures of knee joint size were evaluated in axial CT slices: medio-lateral femur width, antero-posterior lateral condylar height, medio-
\end{abstract}

Peter Wilhelm Ferlic

peter.ferlic@gmail.com

Armin Runer

armin.runer@rolmail.net

Florian Dirisamer

florian.dirisamer@orthopaedie-linz.com

Peter Balcarek

balcarek@sportklinik.de

Johannes Giesinger

johannes.giesinger@i-med.ac.at

Rainer Biedermann

rainer.biedermann@i-med.ac.at

Michael Christian Liebensteiner

michael.liebensteiner@i-med.ac.at

1 Department of Orthopaedic Surgery, Medical University of Innsbruck, Anichstrasse 35, 6020 Innsbruck, Austria

2 Orthopädie \& Sportchirurgie, Linz-Puchenau, Austria

3 Arcus Sportklinik, Pforzheim, Germany

4 Department of Psychiatry, Medical University of Innsbruck, Innsbruck, Austria lateral width of the tibia, width of the patella and the proximaldistal joint size (TT-TE). Furthermore, the length of the femur, the tibia and the total leg length were measured in the CT scanogram. Correlation analysis of TT-TG and the other parameters was done by calculating the Spearman correlation coefficient.

Results In the PFI group lateral condylar height $(r=0.370)$, tibia width $(r=0.406)$ and patella width $(r=0.366)$ showed significant moderate correlations $(p<0.03)$ with TT-TG. Furthermore, we found a significant correlation between TTTG and tibia length $(r=0.371)$ and total leg length $(r=381)$. The control group showed no significant correlation between TT-TG and knee joint size or between TT-TG and measures of lower limb length.

Conclusions Tibial tuberosity-trochlear groove distance correlates with several parameters of knee joint size and leg length in patients with patellofemoral instability. Application of indices determining TT-TG as a ratio of joint size could be helpful in establishing the indication for medial transfer of the tibial tuberosity in patients with PFI.

Level of evidence Level III

Keywords Knee $\cdot$ Patella $\cdot$ Patellofemoral $\cdot$ Patellofemoral instability $\cdot \mathrm{Tt}-\mathrm{TG}$

\section{Introduction}

Patellofemoral instability (PFI) linked to pathological tibial tuberosity-trochlear groove distance (TT-TG) can be surgically addressed by medial transfer of the tibial tuberosity. Dejour et al. described increased TT-TG as one main risk factor for the development of PFI in addition to trochlear dysplasia, quadriceps dysplasia and patella alta [1]. The threshold indicating operative treatment was set at a TT-TG of more than 
$20 \mathrm{~mm}$ [1]. However, this absolute value does not respect the measurements of the individual joints. Hingelbaum et al. hypothesized that a TT-TG distance of more than $20 \mathrm{~mm}$ in a large knee may have a much smaller pathological impact on patellofemoral alignment than in a smaller knee [2]. Therefore, recent literature has introduced various indices evaluating TT-TG as a ratio of joint size: the TT-TG/TT-TE ratio expresses TT-TG in relation to the proximal-distal joint size [2] and the TT-TG/femur width ratio in relationship to the medio-lateral width of the distal femur [3, 4]. Also, Camp et al. studied a patellar instability ratio in order to individualize the TT-TG distance. They reported that TT-TG divided by trochlea width and patella width more effectively predict recurrent instability than the TT-TG distance alone [5].

The mentioned indices are based on the assumption that the pathological impact of TT-TG is influenced by the prevailing proportions of the knee and the patellofemoral joint and that TT-TG positively correlates with the joint size, in other words that an increase in joint size produces an increase in TT-TG. However, three recent studies investigating the correlation between TT-TG and the medio-lateral width of the distal femur did not find such a correlation $[3,4,6]$. Consequently, the questions, whether TT-TG indices should be used instead of absolute TT-TG values, remains to be answered.

Therefore, the aim of the present study was to evaluate the correlation between TT-TG and joint size with a more differentiated three-dimensional approach, while taking into account several different parameters of knee joint size as well as lower limb dimensions in patients with PFI and a control group with stable patellofemoral joints. It was hypothesized that:

1) TT-TG significantly correlates with parameters of joint size in a PFI population (H1)

2) TT-TG significantly correlates with parameters of leg length in a PFI population (H2)

3) TT-TG significantly correlates with parameters of joint size in a control population (H3)

4) TT-TG significantly correlates with parameters of leg length in a control population (H4)

\section{Patients and methods}

After being approved by the ethics committee of our medical university a retrospective analysis of all lower limb CT scans available in our hospital's digital patient archiving system between August 1996 and February 2013 was performed. This covered a total of 560 multislice CTs of lower limbs performed in a helical scan technique, without contrast media. The radiologic examinations with a $2.5-\mathrm{mm}$ slice thickness were conducted at the level of the hips, knees and ankles including a CT scanogram of the lower limbs. The patients were scanned in the supine position with the knee fully extended; $378 \mathrm{CT}$ scans of patients with previous operations or fractures in the region of interest were excluded from this study. Of the remaining 182 cases, all cases with patellofemoral instability were included. These were 36 cases which had a history of at least one patella dislocation and were designated the PFI group. Furthermore, another 30 cases without history of patellofemoral instability were randomly selected from the data base to be included in this study. These 30 cases had deformities, mainly posttraumatic, on one leg and the measurements were done on the contralateral healthy side.

To evaluate the joint proportions five values were measured in axial CT slices (Fig. 1). The medio-lateral femur width and antero-posterior lateral condylar height were measured on the slice $5 \mathrm{~mm}$ distal to the first cut on which the trochlea was fully visible, as described by Biedert and Bachmann [7]. The medio-
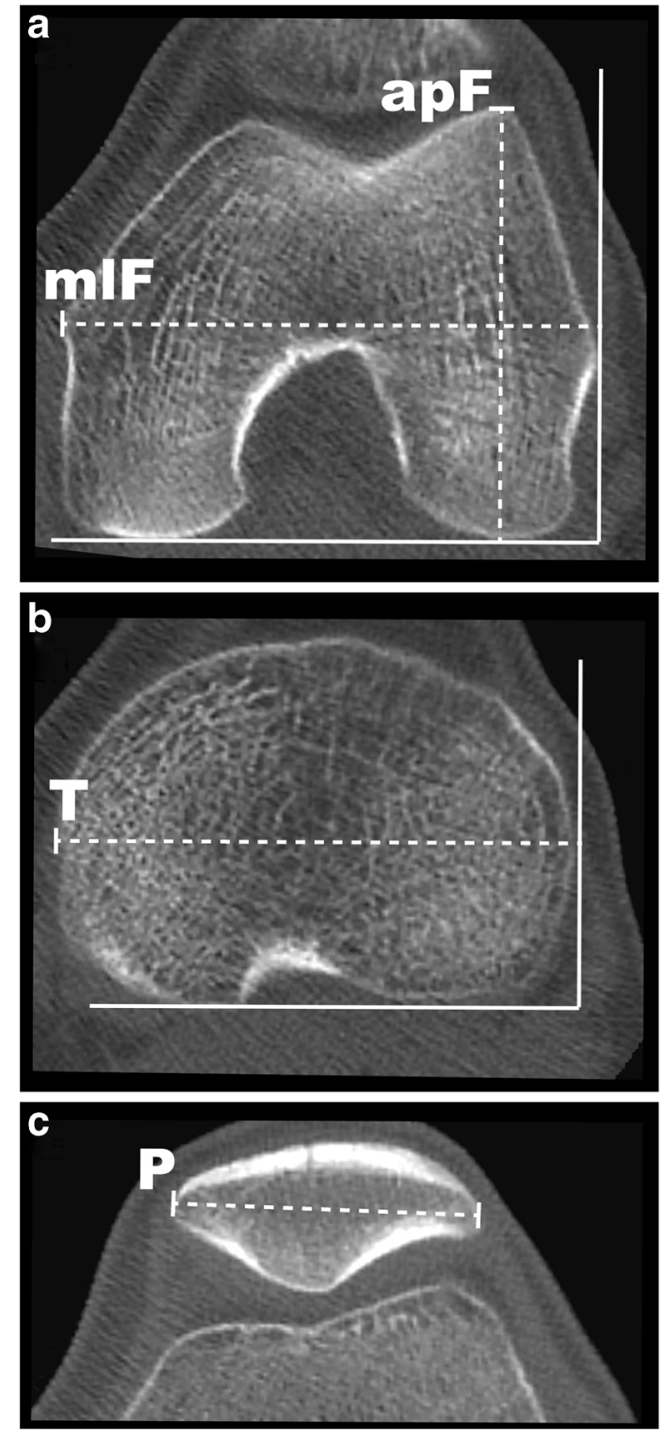

Fig. 1 Measures of joint size on axial CT slices: (a) medio-lateral femur $(\mathrm{mlF})$ width and antero-posterior lateral condylar height $(\mathrm{apF}),(\mathbf{b})$ mediolateral width of the proximal tibia $(\mathrm{T}),(\mathbf{c})$ width of the patella $(\mathrm{P})$ 
lateral width of the tibia was measured in a manner similar to that for the width of the femur on the slice with the greatest tibia width proximal to the tibiofibular joint. The width of the patella was measured along the patella axis in the slice where the patella showed its greatest width. Similar to Hingelbaum et al. [2] the proximal-distal joint size (TT-TE) was determined as the proximal-distal distance between the entrance of the chondral trochlear groove on the first slice where the trochlea was fully visible (TE) and the complete onset of the patella tendon at the tibial tubercle (TT), which was calculated by multiplying the number of slices by the slice thickness.

Furthermore, as a reference for patient size, femur length (FL; distance between the superior aspect of the femoral head and the distal portion of the medial femoral condyle), tibia length (TL; distance between the medial tibial plateau and the tibial plafond) and total leg length (LL; distance between the superior aspect of the femoral head and the tibial plafond) were measured in the CT scanogram (Fig. 2) [8, 9].

To evaluate TT-TG first a tangent to the posterior condylar cortices was drawn. Then a line perpendicular to the posterior condylar line was drawn passing through the deepest point of the trochlear groove on the slice $5 \mathrm{~mm}$ distal to the first cut with full visibility of the trochlea. The trochlea line was transferred to the most cranial slice, on which the patellar tendon was completely in contact with the tibial tubercle. The distance perpendicular from the trochlea line to the middle of the tibial tubercle was measured (TT-TG) $[10,11]$.

All quantitative values were measured by two investigators and the mean was used for further analysis. To evaluate the inter-observer agreement ICC was calculated and graded according to Landis and Koch [12], who characterized values $<0$ as indicating no agreement and $0-0.20$ as slight, $0.21-0.40$ as fair, $0.41-0.60$ as moderate, $0.61-0.80$ as substantial, and $0.81-1$ as almost perfect agreement.

The statistical analysis was performed using SPSS v.18.0 (IBM Corp., USA) and Prism v. 6 (GraphPad Software, CA, USA). The values were tested for normal distribution using the D'Agostino-Pearson normality test. As not all parameters within the subgroups passed this test, further correlation analysis was done with the Spearman correlation coefficient. Correlation strength was defined as follows: $0.0 \leq r \leq 0.2$ : weak correlation; $0.2 \leq r \leq 0.5$ : moderate correlation; $0.5 \leq r \leq 0.8$ : strong correlation; $0.8 \leq r \leq 1.0$ : very strong correlation [13]. The level of significance was set at $p<0.05$. Differences in TTTG and TT-TG indices in the PFI and the control group were assessed with the Kolmogorov-Smirnov test.

\section{Results}

Thirty-six PFI cases (mean age, $24.5 \pm 5.3$ years; range, 14.9 33.9 years) were compared with 30 controls (mean age, $24.8 \pm 8.4$ years; range, $15.1-45.39$ years). The descriptive

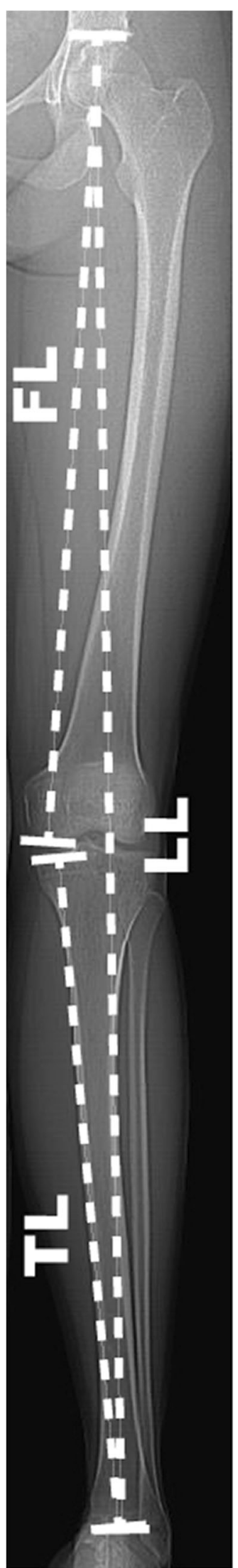

Fig. 2 Measurement of lower limb length in CT scanogram. $F L$ femur length, $T L$ tibia length, $L L$ leg length 
statistics for the parameters investigated are listed in Table 1. The ICC of all radiologic parameters was at least 0.9 and therefore classified as an almost perfect intra-rater agreement, according to the grading of Landis and Koch [12] (Table 1).

In the PFI group lateral condylar height $(r=0.370)$, tibia width $(r=0.406)$ and patella width $(r=0.366)$ showed significant moderate correlations $(p<0.03)$ with TT-TG (Table 2). Furthermore, we found a significant correlation between TT-TG and tibia length $(r=0.371)$ and total leg length $(r=0.381)$.

Analysis of the control group revealed no significant correlation between TT-TG and knee joint size. Similarly, no significant correlations between TT-TG and lower limb length were observed.

Additional exploratory analyses, not linked to our primary hypotheses, showed significantly strong to very strong correlations $(r \geq 0.586, p<0.01)$ among all measures of individual knee joint size in the whole study population. Furthermore, these measurements showed significantly strong to very strong $(r \geq 0.540, p<0.01)$ correlations with the length of the femur, the length of the tibia and total leg length.

We found significant differences in the TT-TG and the TTTG indices between the PFI and the control group (Table 3). The $p$-values for the indices of TT-TG divided by femur width, lateral condylar height, tibia width and patella width were lower than for absolute TT-TG values.

\section{Discussion}

The most important findings made in this study were that in individuals with PFI, TT-TG correlated with several parameters of knee joint size (H1) and with parameters of leg length (H2). In control subjects TT-TG did not show a significant correlation with any of the investigated parameters of joint size (H3) or leg length (H4). Consequently, the study hypothesis was deemed confirmed in the PFI group but not in the control group.

Our observations partially confirm previous studies, but also give new insights into the anatomical features of the knee joint in patients with PFI. Similar to the findings in our control group, three previous studies did not observe any significant correlation between TT-TG and the width of the distal femur $[3,4,6]$. However, Dornacher et al. did not find a correlation between TT-TG and femur width in their PFI group either and therefore concluded that TT-TG has to be considered a very individual parameter in knee realignment surgery [6]. While Pennock et al. and Dornacher et al. assessed the interepicondylar distance as a measure of medio-lateral femur width, Balcarek et al. applied a similar measurement method as used in the present study (Fig. 1) [3, 4, 6]. However, those studies did not assess other parameters of joint size. Only Pennock et al. reported that TT-TG was found to increase as a function of body height $(r=0.14)$ and therefore indicated that there is some evidence for an interaction between TT-TG and individual size [4]. Similarly, we also observed significant correlations between the TT-TG and the length of the tibia as well as the leg.

The present study investigated joint size in three dimensions. Additional to medio-lateral femur width, four other parameters evaluating joint size were also investigated in our study: lateral condylar height, medio-lateral width of the tibia, width of the patella and proximal-distal joint size (TT-TE). By applying this multidimensional approach to the evaluation of individual knee size, the analysis revealed significant correlations between TT-TG and antero-posterior lateral trochlea height, width of the tibia and width of the patella in the PFI group. From our data we cannot support the above-mentioned conclusion drawn by Dornacher et al. [6] and think that individual joint size should be taken into account in realignment surgery of the knee.

Our exploratory analysis showed significant correlations between joint size and leg length as well as between femoral

Table 1 Descriptive statistics of the parameters found in the patellofemoral instability (PFI) and control groups

\begin{tabular}{llll}
\hline Measurement & PFI mean \pm SD (range) & Control mean \pm SD (range) & Interrater agreement (ICC) \\
\hline Medio-lateral femur width & $75.5 \pm 6.6(65.4-88.9)$ & $80.8 \pm 6.4(70.1-94.0)$ & $\mathbf{0 . 9 9 1}^{++}(0.984-0.995)$ \\
Antero-posterior lateral trochlea height & $61.4 \pm 4.8(50.4-72.2)$ & $64.2 \pm 4.4(53.3-71.9)$ & $\mathbf{0 . 9 7 1}^{++}(0.945-0.983)$ \\
Medio-lateral tibia width & $72.4 \pm 6.1(62.9-84.0)$ & $76.8 \pm 5.5(68.6-89.0)$ & $\mathbf{0 . 9 9 2}^{++}(0.974-0.996)$ \\
Patella width & $39.5 \pm 3.7(31.0-45.9)$ & $43.6 \pm 4.0(38.2-52.5)$ & $\mathbf{0 . 9 8 8}^{++}(0.968-0.994)$ \\
Proximal-distal joint size & $49.3 \pm 4.1(40.0-58.8)$ & $52.6 \pm 5.8(42.5-62.5)$ & $\mathbf{0 . 9 1 4}^{++}(0.857-0.948)$ \\
Tibial tuberosity-trochlear groove distance & $16.7 \pm 4.3(9.4-24.6)$ & $12.7 \pm 4.7(4.1-22.2)$ & $\mathbf{0 . 9 0 0}^{++}(0.716-0.954)$ \\
Femur length & $452.3 \pm 28.9(396.0-526.0)$ & $470.7 \pm 26.1(425.5-521.5)$ & $\mathbf{0 . 9 5 1}^{++}(0.921-0.970)$ \\
Tibia length & $359.3 \pm 24.7(302.0-396.0)$ & $371.5 \pm 24.1(318.0-412.0)$ & $\mathbf{0 . 9 9 4}^{++}(0.990-0.996)$ \\
Leg length & $813.9 \pm 50.5(704.0-890.0)$ & $845.7 \pm 48.2(758.0-936.0)$ & $\mathbf{0 . 9 9 6}^{++}(0.994-0.998)$ \\
\hline
\end{tabular}

Values are given in millimeters. Data given as: mean \pm SD (range) and interrater agreement of the radiologic measurements in the whole study population, with data given as: ICC (95\% confidence interval). ICC were graded according to Landis and Koch [12]: $\left({ }^{++}\right)$"almost perfect" 
Table 2 Summary of the correlation analysis between the tibial tuberosity-trochlear groove (TT-TG) distance and parameters describing the joint size in the group of patients with patellofemoral instability (PFI) and the control group

\begin{tabular}{lll}
\hline Measurement & PFI TT-TG & Control TT-TG \\
\hline Medio-lateral femur width & $0.311(p=0.065)$ & $-0.117(p=0.539)$ \\
Antero-posterior lateral trochlea height & $\mathbf{0 . 3 7 0}^{*}(\boldsymbol{p}=\mathbf{0 . 0 2 6})$ & $0.008(p=0.966)$ \\
Medio-lateral tibia width & $\mathbf{0 . 4 0 6}(\boldsymbol{p}=\mathbf{0 . 0 1 4})$ & $-0.131(p=0.489)$ \\
Patella width & $\mathbf{0 . 3 6} *(\boldsymbol{p}=\mathbf{0 . 0 2 8})$ & $-0.239(p=0.203)$ \\
Proximal-distal joint size & $0.163(p=0.342)$ & $-0.202(p=0.284)$ \\
Femur length & $0.325(p=0.053)$ & $0.197(p=0.297)$ \\
Tibia length & $\mathbf{0 . 3 7 1}(\boldsymbol{p}=\mathbf{0 . 0 2 6})$ & $0.152(p=0.423)$ \\
Leg length & $\mathbf{0 . 3 8 1}(\boldsymbol{p}=\mathbf{0 . 0 2 2})$ & $0.239(p=0.204)$ \\
\hline
\end{tabular}

Values represent Spearman correlation coefficient; $p$-values with asterisks mark significant values $(p<0.05)$ and tibial length. This is in accordance with the data of Dornacher et al., who also found a strong correlation between medio-lateral femur width and body size [6]. Furthermore, we observed significant correlations among all parameters used for knee joint size evaluation.

With regard to the question of clinical relevance, the results of this study support the idea of other authors of normalizing TT-TG to individual patient size, as we observed several significant correlations between TT-TG and parameters of joint size and leg length in patients with PFI. It has been reported that surgical treatment significantly reduces the risk of patellar redislocation [14]. An increased tibial TT-TG can be surgically addressed by medial transfer of the tibial tuberosity, and using an index instead of the absolute TT-TG value can be helpful when indicating operative treatment. Similarly, patellar height, which is also an established risk factor for PFI, is assessed with ratios rather than a distance given in millimeters [15]. While Pennock et al. suggested to normalize TT-TG to patient height [4], Hingelbaum et al. recommended to evaluate TT-TG in relation to the proximal-distal joint size [2] and Camp et al. in relation to patella or tibia width [5]. As we found the strongest correlation between TT-TG and tibia width, the ratio of TT-TG divided by tibia width could be a promising parameter for clinical use. Furthermore, tibia width can be easily assessed in CT scans used to measure TT-TG and no further radiologic examination are needed, as it would be the case when evaluating leg length. However, further studies are needed to determine standard values that can be applied as a threshold between physiological variances of TT-TG distance and pathological increased values leading to PFI.

This study has some limitations that deserve mention, which can also be taken as suggestions for future studies. This was a retrospective analysis in which the observer was not blinded to the allocation of patients to the control or the PFI group, as in the PFI group morphologic findings in the knee joint were apparent. Inclusion of clinical outcome data (e.g. patellofemoral questionnaire) would have been of further interest. Using CT scans permitted very accurate evaluation of the bony morphology, which was the main intention of this study. This approach, however, prevented us from investigating other factors influencing the stability of the patellofemoral joint, which is a complex system also comprised of passive (retinacula) and active (muscles) soft tissues. Furthermore, the number of patients included was too small to determine standard values for women and men, that can be applied as a threshold between physiological variances of TT-TG indices and pathological TT-TG indices. Despite these limitations this study provides new scientific knowledge, as for the first time the correlations between TTTG and total leg length as well as length of femur and tibia were determined and a multidimensional approach to knee joint size evaluation was conducted. As significant correlations were found between TT-TG and several parameters of knee joint size, further studies should be done to set up standard values for a TT-TG index that can be used in clinical practice.

Table 3 Differences between tibial tuberosity-trochlear groove (TT-TG) distance and calculated TT-TG indices found in the PFI and the control group

\begin{tabular}{llll}
\hline Measurement & PFI mean \pm SD (range) & Control mean \pm SD (range) & $p$-value \\
\hline TT-TG & $16.7 \pm 4.3(9.4-24.6)$ & $12.7 \pm 4.7(4.1-22.2)$ & $0.16 \pm 0.06(0.05-0.27)$ \\
TT-TG divided by medio-lateral femur width & $0.22 \pm 0.06(0.14-0.33)$ & 0.012 \\
TT-TG divided by antero-posterior lateral trochlea height & $0.27 \pm 0.07(0.17-0.39)$ & $0.07(0.06-0.34)$ \\
TT-TG divided by medio-lateral tibia width & $0.21 \pm 0.06(0.15-0.34)$ & $0.17 \pm 0.06(0.05-0.29)$ \\
TT-TG divided by patella width & $0.42 \pm 0.1(0.25-0.6)$ & $0.3 \pm 0.11(0.08-0.55)$ & $\mathbf{0 . 0 1 1}$ \\
TT-TG divided by proximal-distal joint size & $0.34 \pm 0.09(0.19-0.54)$ & $0.25 \pm 0.09(0.07-0.4)$ & $\mathbf{0 . 0 0 5}$ \\
\hline
\end{tabular}

Data are given as mean $\pm \mathrm{SD}$ (range). $P$-values calculated with Kolmogorov-Smirnov test. P-values in bold text indicate statistical significance 


\section{Conclusion}

Tibial tuberosity-trochlear groove distance correlates with several parameters of knee joint size and leg length in patients with patellofemoral instability. Application of indices determining TT-TG as a ratio of joint size could be helpful in establishing the indication for medial transfer of the tibial tuberosity in patients with PFI.

Acknowledgements Open access funding provided by University of Innsbruck and Medical University of Innsbruck.

\section{Compliance with ethical standards}

Ethical approval All procedures performed in studies involving human participants were in accordance with the ethical standards of the institutional and/or national research committee and with the 1964 Helsinki Declaration and its later amendments or comparable ethical standards. For this type of study formal consent is not required.

Conflict of interest The authors declare that they have no conflict of interest.

Open Access This article is distributed under the terms of the Creative Commons Attribution 4.0 International License (http:// creativecommons.org/licenses/by/4.0/), which permits unrestricted use, distribution, and reproduction in any medium, provided you give appropriate credit to the original author(s) and the source, provide a link to the Creative Commons license, and indicate if changes were made.

\section{References}

1. Dejour H, Walch G, Nove-Josserand L, Guier C (1994) Factors of patellar instability: an anatomic radiographic study. Knee Surg Sports Traumatol Arthrosc 2(1):19-26

2. Hingelbaum S, Best R, Huth J, Wagner D, Bauer G, Mauch F (2014) The TT-TG index: a new knee size adjusted measure method to determine the TT-TG distance. Knee Surg Sports Traumatol Arthrosc 22(10):2388-2395. doi:10.1007/s00167-014-3204-1

3. Balcarek P, Jung K, Frosch KH, Sturmer MK (2011) Value of the tibial tuberosity-trochlear groove distance in patellar instability in the young athlete. Am J Sports Med 39(8):1756-1761. doi:10. 1177/0363546511404883

4. Pennock AT, Alam M, Bastrom T (2014) Variation in Tibial tubercle-trochlear groove measurement as a function of age, sex, size, and patellar instability. Am J Sports Med 42(2):389-393. doi: 10.1177/0363546513509058

5. Camp CL, Heidenreich MJ, Dahm DL, Stuart MJ, Levy BA, Krych AJ (2016) Individualizing the tibial tubercle-trochlear groove distance: patellar instability ratios that predict recurrent instability. Am J Sports Med 44(2):393-399. doi:10.1177/0363546515602483

6. Dornacher D, Reichel H, Kappe T (2016) Does tibial tuberositytrochlear groove distance (TT-TG) correlate with knee size or body height? Knee Surg Sports Traumatol Arthrosc 24(9):2861-2867. doi:10.1007/s00167-015-3526-7

7. Biedert RM, Bachmann M (2009) Anterior-posterior trochlear measurements of normal and dysplastic trochlea by axial magnetic resonance imaging. Knee Surg Sports Traumatol Arthrosc 17(10): 1225-1230. doi:10.1007/s00167-009-0824-y

8. Sabharwal S, Kumar A (2008) Methods for assessing leg length discrepancy. Clin Orthop Relat Res 466(12):2910-2922. doi:10. 1007/s11999-008-0524-9

9. Vaidya R, Anderson B, Elbanna A, Colen R, Hoard D, Sethi A (2012) CT scanogram for limb length discrepancy in comminuted femoral shaft fractures following IM nailing. Injury 43(7):11761181. doi:10.1016/j.injury.2012.03.022

10. Camp CL, Stuart MJ, Krych AJ, Levy BA, Bond JR, Collins MS, Dahm DL (2013) CT and MRI measurements of tibial tubercletrochlear groove distances are not equivalent in patients with patellar instability. Am J Sports Med 41(8):1835-1840. doi:10.1177/ 0363546513484895

11. Koëter S, Horstmann WG, Wagenaar F-CBM, Huysse W, Wymenga AB, Anderson PG (2007) A new CT scan method for measuring the tibial tubercle trochlear groove distance in patellar instability. Knee 14(2):128-132. doi:10.1016/j.knee.2006.11.003

12. Landis JR, Koch GG (1977) The measurement of observer agreement for categorical data. Biometrics 33(1):159-174. doi:10.2307/ 2529310

13. Cohen J (1988) Statistical power analysis for the behavioral sciences, vol 2nd. L. Erlbaum Associates, Hillsdale. doi:10.1234/ 12345678

14. Saccomanno MF, Sircana G, Fodale M, Donati F, Milano G (2016) Surgical versus conservative treatment of primary patellar dislocation. A systematic review and meta-analysis. Int Orthop 40(11): 2277-2287. doi:10.1007/s00264-015-2856-x

15. van Duijvenbode D, Stavenuiter M, Burger B, van Dijke C, Spermon J, Hoozemans M (2016) The reliability of four widely used patellar height ratios. Int Orthop 40(3):493-497. doi:10. 1007/s00264-015-2908-2 